\title{
Disconnection of the Amygdala from Visual Association Cortex Impairs Visual Reward-Association Learning in Monkeys
}

\author{
E. A. Gaffan, David Gaffan, ${ }^{1}$ and Susan Harrison ${ }^{1}$ \\ Department of Psychology, Reading University, Reading RG6 2AL, and 'Department of Experimental Psychology, Oxford \\ University, Oxford OX1 3UD, England
}

\begin{abstract}
Cynomolgus monkeys (Macaca fascicularis) were trained in a task that assessed their ability to associate visual stimuli with food reward. Acquisition of stimulus-reward associations was measured under 2 conditions, a 2-stimuli acquisition condition and a 1-stimulus acquisition condition. On each trial in the 2-stimuli condition, the positive (correct) and negative (incorrect) stimuli were presented side by side and the animal chose one by touching it; if the choice was correct, a food reward was dispensed. On each trial in the 1-stimulus condition, elther the positive or the negative stimulus was presented alone; if the stimulus was the positive, it was followed by reward delivery, regardless of the animal's response to it, and if it was the negative, it was not followed by reward delivery. Thus, reward delivery was contingent upon the animal's response to the stimuli in the 2-stimuli condition but not in the 1-stimulus condition. The effect of acquisition trials under these 2 conditions was measured, in both conditions, by the animal's subsequent choice when presented with the 2 stimuli side by side. Following preoperative training in this task, the animals were first subjected to unilateral ablation of the inferotemporal cortex. This operation had little effect on the animals' learning ability. Then, the amygdala was ablated in the hemisphere contralateral to that in which the unilateral inferotemporal ablation had been carried out. This combination of crossed unilateral lesions of the amygdala and of the inferotemporal cortex, which disconnects the amygdala from the output of visual association cortex, produced a profound impairment in stimulusreward-associative learning. The severity of impairment was equal in the 2 acquisition conditions. These results contrast with those of a previous experiment on the ability to associate visual stimuli with an auditory secondary reinforcer, not with food reward. The amygdala has a specific role in the learning of associations with primary reward, both when those associations are response-contingent and when they are not.
\end{abstract}

Bilateral amygdalectomy in monkeys has sometimes, but not always, been reported to impair visual discrimination learning that is motivated by food reward. Reviewing this contradictory literature, Gaffan and Harrison (1987) pointed out that the dis-

Received May 19, 1987; revised Dec. 15, 1987; accepted Dec. 15, 1987.

This research was supported by the Medical Research Council.

Correspondence should be addressed to either E. A. Gaffan, Department of Psychology, Reading University, Whiteknights, Reading RG6 2AL, England; or D. Gaffan, Department of Experimental Psychology, South Parks Road, Oxford OX1 3UD, England.

Copyright $\odot 1988$ Society for Neuroscience $0270-6474 / 88 / 093144-07 \$ 02.00 / 0$ criminative stimuli in a learning task might be associated with a variety of different types of attribute of the reward event. When the positive discriminative stimulus is paired with a primary reinforcer such as a peanut, for example, the stimulus might be associated either with the intrinsically rewarding attributes of the peanut, such as its taste, or with attributes of the peanut that are not intrinsically rewarding, such as its visual appearance. To investigate the role of the amygdala in learning about different attributes of the reward event, Gaffan and Harrison (1987) examined a task in which the reward event that sustained within-problem visual discrimination learning was an auditory secondary reinforcer. In that task, the auditory secondary reinforcer was associated with the delivery of a primary food reinforcer, but the visual discriminanda were associated only with the auditory reinforcer and not with food. Thus, the visual stimuli were not associated with the intrinsically rewarding attributes of food; instead, they were associated with an auditory stimulus that had acquired an extrinsic reward value by virtue of its own association with the intrinsically rewarding attributes of food. Gaffan and Harrison compared the effects on this task of disconnecting the amygdala either from visual association cortex or from auditory association cortex. These disconnections were accomplished by crossed unilateral lesions of the amygdala in one hemisphere and of association cortex in the opposite hemisphere, and by forebrain commissurotomy. Disconnection of the amygdala from the auditory modality produced a profound impairment in the learning task, but disconnection of the amygdala from the visual modality left learning unimpaired. The hypothesis was put forward that the amygdala is involved in associating stimuli with the intrinsically reinforcing attributes of reward events, and not in associating stimuli with the attributes that are intrinsically motivationally neutral. The present cxperiment was designed as a further test of this hypothesis and as an investigation of an alternative interpretation of Gaffan and Harrison's (1987) findings.

One simple prediction from Gaffan and Harrison's hypothesis is that disconnection of the amygdala from the visual modality should impair visual discrimination learning in tasks where the visual discriminative stimuli are associated with food reward. Therefore, in the present experiment we used the same apparatus and the same discriminative stimuli as in Gaffan and Harrison's experiment, but the positive discriminanda were associated with food, rather than with a secondary reinforcer. We disconnected the amygdala from visual association cortex in the same way as in the earlier study, by crossed unilateral lesions of the visual association cortex in one hemisphere and of the amygdala in the opposite hemisphere. The prediction from the hypothesis outlined above was that this disconnection should produce an 
impairment in the present task, although it produced no impairment in Gaffan and Harrison's (1987) task.

The present task was also designed to test an alternative interpretation of Gaffan and Harrison's (1987) findings. In their task, the association between the auditory secondary reinforcer and the food reward was not contingent upon the animal's behavior. The auditory secondary reinforcer was followed by the delivery of food on some trials, according to the schedule described in detail by Gaffan and Harrison, but the delivery of food was not determined by the animal's behavior in the presence of the auditory secondary reinforcer. By contrast, the associations between the positive visual discriminanda and the auditory secondary reinforcer in their task depended on the animal's behavior in the presence of the visual stimuli: The positive and negative visual stimuli in any discrimination problem in their task were presented side by side, and only if the animal chose the positive stimulus by touching it was the secondary reinforcer produced. Thus, the associations between the visual discriminanda and the auditory secondary reinforcer were response-contingent, but the association between the auditory secondary reinforcer and food was not. It is possible that this difference underlay the lesion effects that Gaffan and Harrison observed; for example, it is possible that the amygdala is selectively involved in interstimulus associative learning, and not in instrumental learning of associations that are contingent upon the animal's behavior. Therefore, the present experiment examined acquisition of stimulus-food associations under 2 conditions: one in which the stimulus-food associations were response-contingent, and one in which they were not.

The 2 acquisition conditions in the present experiment, one that is response-contingent and the other not, are 2-stimuli and 1 -stimulus acquisition conditions. In the 2-stimuli acquisition condition, the positive and negative visual discriminative stimuli are presented side by side; the animal chooses one by touching it, and if the chosen stimulus is the positive stimulus, a food reward is dispensed. Thus, the relation between the positive visual stimulus and the food in this condition of the present task is identical to the relation between the positive visual stimulus and the auditory secondary reinforcer in Gaffan and Harrison's (1987) task. In the 1 -stimulus condition, the animal produces a single visual stimulus by touching the screen on which stimuli are displayed. Whether the stimulus produced is the positive or the negative is determined at random. If it is the positive, it is followed by food reward. But the delivery of food in this condition is independent of the animal's behavior in the presence of the discriminative stimuli. Thus, the relation between the positive visual stimulus and the food in this acquisition condition is similar to the relation between the auditory secondary reinforcer and food in Gaffan and Harrison's (1987) task.

Following each of thesc acquisition conditions, stimulus-rcward association was assessed by the animal's choice between the positive and negative stimuli. This retention test is operationally identical to the 2-stimuli acquisition condition. In effect, therefore, the animal is given a series of 2-choice visual discriminations, and some of them are preceded by 1 -stimulus acquisition trials in which the stimulus-reward contingency is independent of the animal's behavior. The number of such 1 -stimulus acquisition trials varies from problem to problem. By examining the effects of varying numbers of 1-stimulus acquisition trials upon the animal's subsequent choice between the stimuli, one can derive a learning curve for the acquisition of stimulus-reward associations in the 1-stimulus acquisition condition and compare it with the learning curve in the 2 -stimuli acquisition condition.

In this task we also recorded the animal's behavior in response to single stimuli in the 1-stimulus acquisition condition. As explained, this behavior was without effect. But it was expected that some responses to the single stimuli would be made through "autoshaping" and "superstition" (Gamzu and Schwam, 1974).

Following preoperative training in this task, the inferotemporal area was ablated unilaterally and the animals were retested to confirm that the unilateral ablation had little effect by itself. Then, the amygdala was ablated unilaterally in the hemisphere contralateral to the inferotemporal ablation. The inferotemporal ablation was the same as that studied by Gaffan and Harrison (1987). This ablation includes unilaterally a cortical ablation that, if made bilaterally, produces a severe impairment in visual associative lcarning for food reward with the present stimulus material (D. Gaffan et al., 1986; E. A. Gaffan et al., 1986).

\section{Materials and Methods}

Subjects. Three male cynomolgus monkeys (Macaca fascicularis) took part in the experiment. At the beginning of preoperative training, as described below, they were experimentally naive. At the time of the first surgery, their average weight was $4.8 \mathrm{~kg}$.

Surgery. There were 2 stages of surgery, the first to ablate the inferotemporal area unilaterally and the second to ablate the amygdala unilaterally. The anesthetic and aseptic methods were as described previously (Gaffan et al., 1984). After each stage of surgery, 17-20 d were allowed for recovery before training resumed.

The unilateral inferotemporal removal was carried out in an identical manner to that in parallel experiments on visual disconnection (Gaffan and Harrison, 1987, 1988). The zygoma was removed and a craniotomy was opened with drill and rongeurs. The dura was cut in a $Y$ shape over the intended area of the ablation, and was sewn afterwards. The ablation was carried out by cauterization and aspiration, with a 19-gauge metal aspirator insulated except at the tip. It included the inferior bank of the superior temporal sulcus, the lateral bank of the occipitotemporal sulcus, and all the cortex between those 2 limits. The parahippocampal gyrus, medial to the occipitotemporal sulcus, was left intact. Anteriorly, the ablation was continued to the tip of the superior temporal sulcus, and the medial boundary in the anterior extent of the lesion was a continuation of the line of the occipitotemporal sulcus. Posteriorly, the ablation was extended into the anterior bank of the lunate sulcus, and was limited by the level of the posterior tip of the lateral sulcus superiorly (although the ablation did not include the lateral sulcus, being bounded still by the inferior bank of the superior temporal sulcus). Both banks of the ascending tip of the inferior occipital sulcus were included in the posterior part of the ablation, as were both banks of the anterior middle temporal sulcus in the anterior part.

The amygdalectomy was carried out in the same way as in the experiment of Gaffan and Harrison (1987). A craniotomy was opened over the frontal lobe and was cxtcnded with rongcurs anteriorly to the brow, medially within $2-3 \mathrm{~mm}$ of the midline, posteriorly to the level of the arcuate sulcus, and laterally and inferiorly into the lateral wall of the temporal fossa to the level of the superior temporal sulcus. The dura was cut along the superior, posterior, and inferior margins of the craniotomy, and turned back in a flap over the orbit and brow. The frontal lobe was gently retracted with a brain spoon to expose the medial surface of the anterior part of the temporal lobe. A 20-gauge metal aspirator that was insulated except at the tip was used to cauterize and remove a patch of pia mater, 2-3 mm wide, on the medial surface of the temporal lobe superior and posterior to the tip of the rhinal sulcus. The amygdala was then ablated through the defect in the pia mater by aspiration with the same metal aspirator. The lateral ventricle and the anterior surface of the hippocampus were visible posterior limits of the lesion. Laterally, gray matter of the amygdala was removed until white matter of the temporal stalk and uncinate fascicle appeared. Inferiorly and anteriorly, the intention was to leave perirhinal and polar cortex intact, as far as possible. The dura was sewn and the wound was closed in layers.

Histology. The animals were given a lethal dose of anesthetic and then perfused through the heart with saline, followed by Formol-saline 
solution. The brains were blocked in the coronal stereotaxic plane posterior to the lunate sulcus and were then removed from the head, photographed, and allowed to sink in a sucrose formalin solution. The brains were cut in $50 \mu \mathrm{m}$ sections on a freezing microtome. Every fifth section was retained and stained with cresyl violet.

Apparatus and stimuli. The computer-controlled apparatus, described in detail by Gaffan et al. (1984), displayed complex patterns singly or in pairs against a plain gray background on a television that the monkeys viewed through a glass screen. The stimuli were presented either in the center of the screen or $90 \mathrm{~mm}$ to the left or right of center. Infrared beams crossing the surface of the glass screen served to detect the animals' touching the stimuli. Each of the 2 visual stimuli that constituted a discrimination problem was made of a small shape superimposed on a larger shape (approximate heights, 20 and $40 \mathrm{~mm}$, respectively). Each of the shapes was taken at random from a predefined set of 127 symbols, and was displayed in a color chosen at random from 255 possibilities. The random number generator that determined stimuli for each problem and left-right position at every trial was seeded with the session number, so the stimulus sequence was unique to each session but the same, in a given numbered session, for all monkeys.

Rewards, consisting of halved blanched peanuts or sugar-coated grains of puffed rice, were dispensed into a bowl just in front of the lower edge of the screen; the automatic dispenser made a $0.35 \mathrm{sec}$ buzz when it operated. The only visible illumination in the experimental cubicle came from the television screen, but an infrared floodlight allowed the monkey to be watched over closed-circuit tclcvision.

Preoperative training. Before training in the main task as described below, the animals were initially trained as follows: Preliminary "shaping" to touch stimuli on the screen was accomplished by the schedules described in detail by Gaffan et al. (1984). The subjects then had 4050 sessions of 2-choice visual discrimination training for immediate food reward. A new pair of stimuli, $S+$ and $S-$, was generated for each discrimination problem. For each trial within a problem, either $\mathbf{S}+$ was displayed on the left and $\mathrm{S}-$ on the right, or vice versa. The stimuli remained on the screen until the animal touched one. If it was $\mathrm{S}+$, a food reward was dispensed. When the animal touched one stimulus, the stimulus that it had not touched disappeared from the screen; the touched stimulus disappeared $0.5 \mathrm{sec}$ after it had been touched. An intertrial interval, during which the screen was blank gray, then commenced, and the next trial began when $8 \mathrm{sec}$ had elapsed without the screen's being touched. For all animals, each of the last 10 sessions of this stage of training consisted of 5 new discrimination problems presented successively for 20 trials each. Then, all animals had 13 sessions of training in a task that was almost identical to the main task described below. Finally, preoperative training was completed with 20 sessions of training in the main task, exactly as described below.

Main task. In every session there were 8 successively presented discrimination problems, each having a newly chosen $\mathbf{S}+$ and $\mathbf{S}-\mathbf{S}+$ and $\mathrm{S}-$ might be displayed either simultaneously in the left and right positions, with the choice of $\mathrm{S}+$ being rewarded, or singly in the center position, in which case $S+$ was terminated by reward delivery and $S-$ was not, regardless of the animal's response. These are the 2-stimuli and 1-stimulus trials described in detail below. Every problem was presented for a total of 14 trials. There were 4 possible sequences of trials within a problem: (1) fourteen 2-stimuli trials, (2) two 1-stimulus trials followed by twelve 2-stimuli trials, (3) four 1-stimulus trials followed by ten 2-stimuli trials, and (4) eight 1 -stimulus trials followed by six 2-stimuli trials. Each of these sequences was followed in 2 of the 8 problems, chosen at random, in each session. For the 1 -stimulus trials, either $\mathbf{S}+$ or $\mathbf{S}-$ was presented at random. For the 2 -stimuli trials, $\mathbf{S}+$ was either on the left or on the right at random.

Each 1-stimulus trial commenced with an "observing stimulus," a white vertical line $24 \mathrm{~mm}$ long at the center of the screen. As soon as the monkey touched the observing stimulus, it was replaced by the scheduled discriminative stimulus, $\mathbf{S}+$ or $\mathbf{S}-$, which remained on the screen for $3.5 \mathrm{sec}$. If it was $\mathrm{S}+$, food was delivered $0.5 \mathrm{sec}$ before the stimulus ended, but if $\mathrm{S}-$, no food was delivered. In either case, the termination of the stimulus was followed by a $5 \mathrm{sec}$ intertrial interval, during which the screen was gray, and any response postponed the next trial for a further $5 \mathrm{sec}$.

The animal's responses to a singly presented $\mathrm{S}+$ and $\mathrm{S}-$ had no effect, but were counted during the first 3 sec of each stimulus (except for the first $0.04 \mathrm{sec}$ after the observing stimulus disappeared, when any beam interruption was assumed to be the residue of the observing response). The criterion of a response was at least $0.02 \mathrm{sec}$ continuous interruption of the beams at the center of the screen. Then, for one more response to be accumulated, there first had to be at least $0.02 \mathrm{sec}$ during which the beams were uninterrupted. The cumulative duration of beam interruption was also recorded for each trial.

For each 2-stimuli trial within a problem, either $\mathbf{S}+$ was displayed on the left and $\mathrm{S}$ - on the right, or vice versa. The stimuli remained on the screen until the animal touched one. If the stimulus touched was $\mathrm{S}+$, a food reward was immediately dispensed. As soon as the animal touched one stimulus, both stimuli disappeared from the screen. An intertrial interval, during which the screen was blank gray, then commenced, and the next trial began when $8 \mathrm{sec}$ had elapsed without the screen's being touched.

Procedure. Following initial preoperative training as described above, 20 sessions of training in the main task completed preoperative training. The inferotemporal cortex was then ablated unilaterally, and 20 further sessions of training in the main task were given. The amygdala was then ablated in the hemisphere contralateral to the inferotemporal ablation, and 20 further sessions of training in the main task completed the experiment.

\section{Results}

\section{Histological}

Microscopic examination of each brain showed that the lesions wcre as intended (see Materials and Methods). The ablation of visual association cortex in the hemisphere contralateral to the amygdalectomy was very similar in all 3 monkeys, and was the same as in our previous experiment with this lesion (see Figs. 1-3 in Gaffan and Harrison, 1987). This lesion included the lateral bank of the occipitotemporal sulcus and the inferior bank of the superior temporal sulcus, but left the superior temporal gyrus and the parahippocampal gyrus intact. Anteriorly, as illustrated in Figure 1, the lesion included both banks of the anterior middle temporal sulcus, but left intact the cortex medial to the anterior middle temporal sulcus. Figure 1 also illustrates the amygdalectomy, in the hemisphere contralateral to the ablation of visual association cortex. It can be seen that the amygdalectomy was complete except for some parts of the posterior dorsal amygdaloid complex. The other 2 monkeys were similar to the monkey illustrated.

Monkey XVA-3 was chosen for illustration (Fig. 1) because this was the monkey with the worst postoperative performance (Table 1); this brain can be compared with the brain of the individual monkey illustrated in an earlier report (see Fig. 1 in Gaffan and Harrison, 1987) that showed the best postoperative performance in the earlier experiment. Comparison indicates that the difference between the unimpaired performance in the earlier experiment and the severe deficit in the present experiment cannot be ascribed to any obvious variation in the extent of the ablations. Microscopic examination and comparison of the 6 brains from the 2 experiments confirmed this conclusion.

\section{Choices in 2-stimuli trials}

As explained in the introduction, 2-stimuli trials played 2 roles in the present task, namely, as one of the acquisition conditions for stimulus-reward associations, and as a test for the retention of those associations from previous acquisition trials. Table 1 shows the accuracy of choice in 2-stimuli trials as a function of the preceding acquisition trials, themselves either 1-stimulus or 2-stimuli trials. As described above (see Main task in Materials and Methods), new discrimination problems had trial sequences of 4 possible types: (1) fourteen 2-stimuli trials, (2) two 1-stimulus trials followed by twelve 2 -stimuli trials, (3) four 1 -stimulus trials followed by ten 2 -stimuli trials, and (4) eight 1 -stimulus trials followed by six 2 -stimuli trials. To explain the derivation 

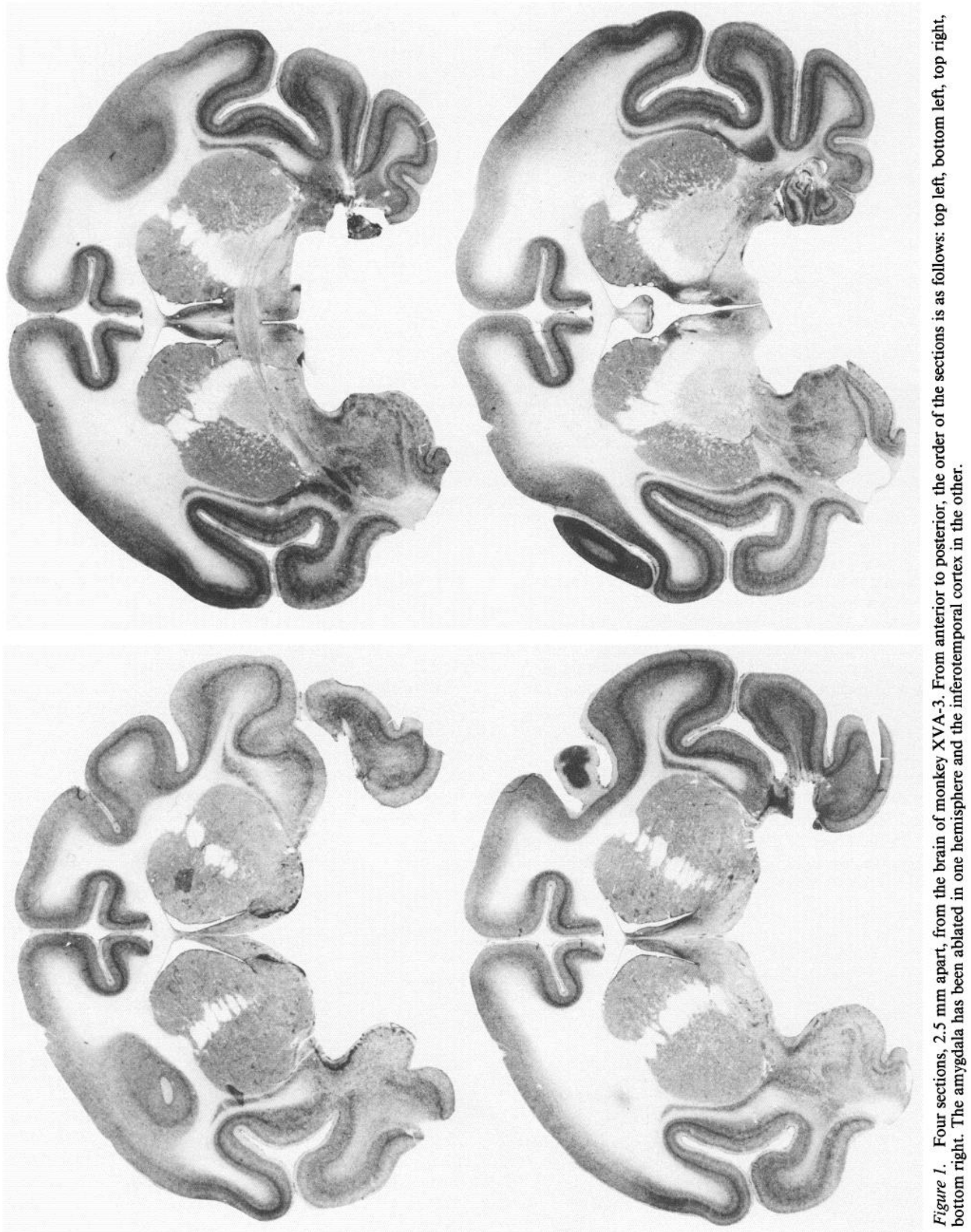


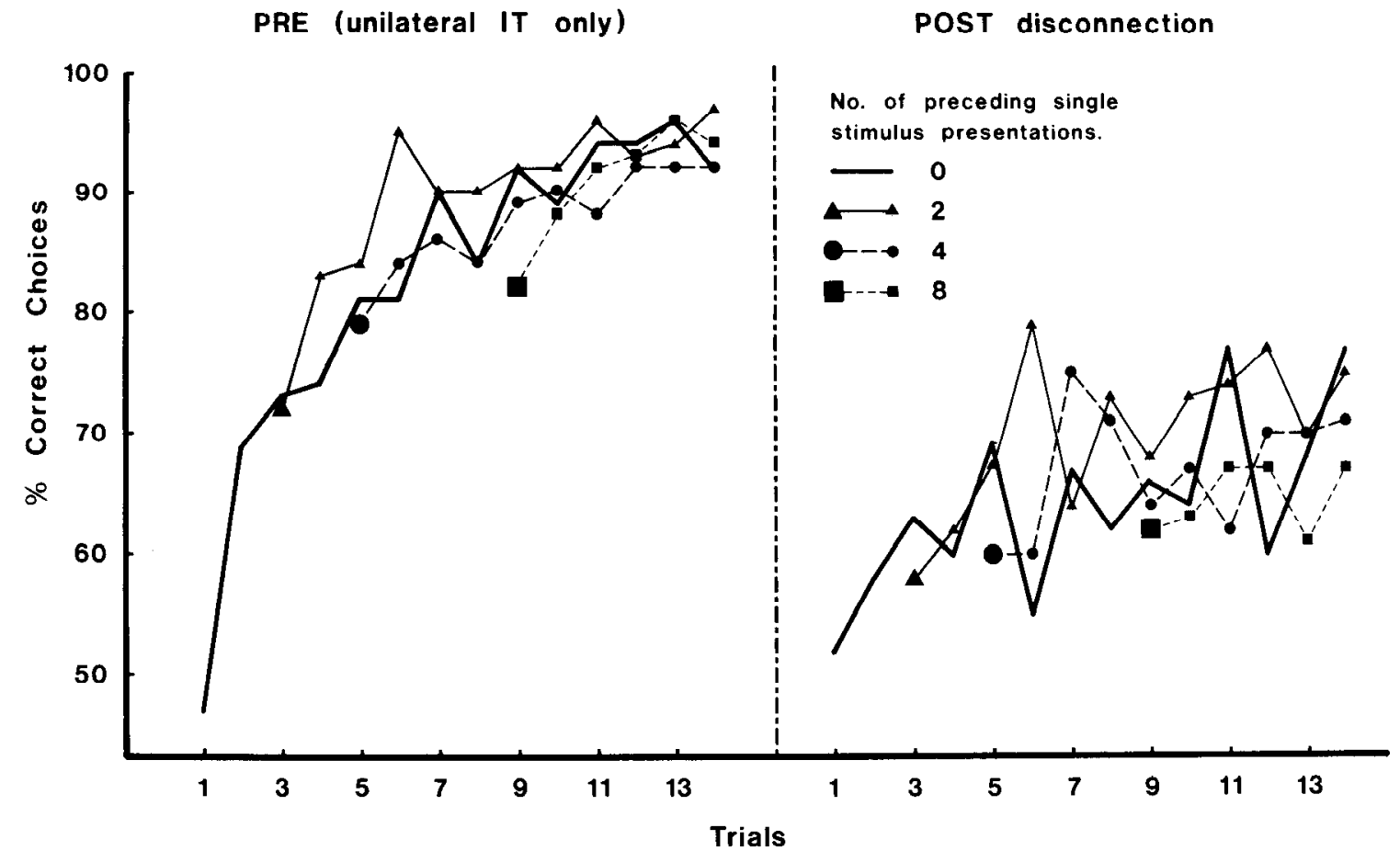

Figure 2. Group learning curves following unilateral inferotemporal ablation (left) and the addition of amygdalectomy in the hemisphere contralateral to the inferotemporal ablation (right). The 4 curves in each panel represent performance at choice trials in 4 types of problem, each having a different number of 1 -stimulus acquisition trials preceding the 2-stimuli choice trials.

of the numbers in Table 1 , consider the 6 numbers that represent monkey XVA-1's preoperative performance. The top row (75.0, $87.5,100.0$ ), representing performance after two, four, and eight 1 -stimulus acquisition trials, is the percentage of correct choices on trial 3 of type 2 problems, trial 5 of type 3 problems, and trial 9 of type 4 problems, respectively. The bottom row $(85.0$, $90.0,100.0$ ), representing performance after two, four, and eight 2-stimuli acquisition trials, is the percentage of correct choices on trials 3,5 , and 9 of type 1 problems.

Table 1 shows that the animals increasingly tended to choose $\mathrm{S}+$ in 2 -stimuli trials as the number of preceding acquisition trials increased. The effect of 1-stimulus acquisition trials was almost equal to the effect of 2 -stimuli acquisition trials. The effects of surgery were straightforward. The first stage, unilateral removal of the inferotemporal cortex, produced little change in performance of the task. The disconnection resulted in a severe disruption that appeared to affect learning from single and choice trials to a similar degree. This is illustrated in more detail in Figure 2, which shows trial-by-trial learning curves from the 4 types of problem, averaged across subjects, in the last 2 phases of the experiment. On inspection, it appears that, following disconnection, the animals continued to show some learning from both singlc and choice trials, and to a similar extent from each, but at a strikingly lower level than before.

Statistical analysis, applied to the data of Table 1, confirmed these impressions. Taking all three phases of the experiment together, there was a significant increase in correct choices as the number of preceding acquisition trials increased from 2 to

\begin{tabular}{|c|c|c|c|c|c|c|c|c|c|c|}
\hline \multirow[b]{3}{*}{ Monkey } & \multirow[b]{3}{*}{ Condition } & \multicolumn{9}{|c|}{ Surgical stage of the experiment } \\
\hline & & \multicolumn{3}{|c|}{$\begin{array}{l}\text { Preoperative } \\
\text { (no. of acquisition } \\
\text { trials) }\end{array}$} & \multicolumn{3}{|c|}{$\begin{array}{l}\text { Unilat. IT } \\
\text { (no. of acquisition } \\
\text { trials) }\end{array}$} & \multicolumn{3}{|c|}{$\begin{array}{l}\text { Disconnection } \\
\text { (no. of acquisition } \\
\text { trials) }\end{array}$} \\
\hline & & 2 & 4 & 8 & 2 & 4 & 8 & 2 & 4 & 8 \\
\hline \multirow[t]{2}{*}{ XVA-1 } & 1-stimulus & 75.0 & 87.5 & 100 & 75.0 & 97.5 & 100 & 60.0 & 67.5 & 80.0 \\
\hline & 2-stimuli & 85.0 & 90.0 & 100 & 82.5 & 90.0 & 97.5 & 72.5 & 80.0 & 80.0 \\
\hline \multirow[t]{2}{*}{ XVA-2 } & 1-stimulus & 72.5 & 85.0 & 75.0 & 75.0 & 70.0 & 72.5 & 67.5 & 60.0 & 52.5 \\
\hline & 2-stimuli & 72.5 & 82.5 & 97.5 & 70.0 & 72.5 & 95.0 & 52.5 & 65.0 & 67.5 \\
\hline \multirow[t]{2}{*}{ XVA-3 } & 1-stimulus & 67.5 & 90.0 & 95.0 & 67.5 & 70.0 & 75.0 & 47.5 & 52.5 & 55.0 \\
\hline & 2-stimuli & 80.0 & 77.5 & 95.0 & 67.5 & 80.0 & 85.0 & 65.0 & 62.5 & 50.0 \\
\hline \multirow[t]{2}{*}{ Average } & 1-stimulus & 71.7 & 87.5 & 90.0 & 72.5 & 79.2 & 82.5 & 58.3 & 60.0 & 62.5 \\
\hline & 2-stimuli & 79.2 & 87.5 & 90.0 & 73.3 & 80.8 & 92.5 & 63.3 & 69.2 & 65.8 \\
\hline
\end{tabular}


Table 2. Frequency per trial of touching stimuli in 1-stimulus trials

\begin{tabular}{lllll} 
& & \multicolumn{3}{c}{ Surgical stage of the experiment } \\
\cline { 3 - 5 } Monkey & Stimulus & $\begin{array}{l}\text { Pre- } \\
\text { operative }\end{array}$ & Unilat. IT & $\begin{array}{l}\text { Discon- } \\
\text { nection }\end{array}$ \\
\hline XVA-1 & S+ & 12.9 & 12.0 & 10.9 \\
& S- & 7.6 & 5.2 & 5.3 \\
XVA-2 & S+ & 3.6 & 4.0 & 3.8 \\
& S- & 3.8 & 4.6 & 4.0 \\
XVA-3 & S+ & 1.9 & 1.1 & 0.5 \\
& S- & 1.8 & 0.9 & 0.4 \\
\hline
\end{tabular}

$8(F=16.32, d f(2,4) ; p<0.05)$. Also, there were significantly more correct choices following 2-stimuli acquisition trials than after 1-stimulus acquisition trials $(F=184.69, d f(1,2) ; p<0.01)$. But the effects of the number of acquisition trials $(2,4$, and 8$)$ and of the type of trial (1-stimulus and 2-stimuli) did not interact with each other $(F<1)$.

There was a significant difference in overall performance across the three surgical stages of the experiment $(F=31.54, d f(2,4)$; $p<0.01)$. Planned comparisons indicated that the small drop in average performance after the unilateral inferotemporal lesion did not reach statistical significance $(F=2.16, d f(1,4) ; p$ $>0.05)$, but the further deterioration following the addition of the amygdala ablation was highly significant $(F=31.54, d f(1,4)$; $p<0.01)$. There was no evidence that this effect of disconnection applied differentially to learning from 1-stimulus and from 2-stimuli trials: The interaction between the effect of the 3 surgical stages and the effect of the 2 types of acquisition trial was not significant $(F<1)$. The interaction between the stages of surgery and the number of preceding trials $(2,4$, or 8$)$ approached significance $(F=3.59, d f(4,8) ; 0.05<p<0.10)$, reflecting the flattening of the learning curves in the final stage.

\section{Comparison with auditory secondary reinforcement}

One of the main purposes of the present experiment, as explained in the introduction, was to compare the present task with the task of Gaffan and Harrison (1987), which required association of visual stimuli with an auditory secondary reinforcer, rather than with food. In both experiments, the animals were tested both as normal animals preoperatively and following disconnection. In the earlier experiment, learning was assessed by performance on trial 4 of discrimination problems; as explained in the earlier paper, there was a special reason, in the design of the task, for the selection of this measure. Furthermore, to allow for the possibility of recovery with practice, the lesion effects in the earlier experiment were assessed from the final 5 sessions of the 20 sessions of training that followed each surgical stage of the experiment. Therefore, to compare the present results with the earlier results, we computed performance on trial 4 of discrimination problems in the last 5 sessions of the preoperative training and in the last 5 sessions of the training that followed disconnection. (This measure is an average of trial 4 performances in problems with trial sequences numbered 1 and 2 in Materials and Methods; these are the problems in which trial 4 was a 2-stimuli choice trial.) The comparison is shown in Figure 3. The effect of the disconnection was quite different in the 2 tasks, as confirmed by a significant interaction, in the data in Figure 3, between disconnection (Pre-op vs Post-op) and the tasks $(F=42.59, d f(1,4) ; p<0.01)$.

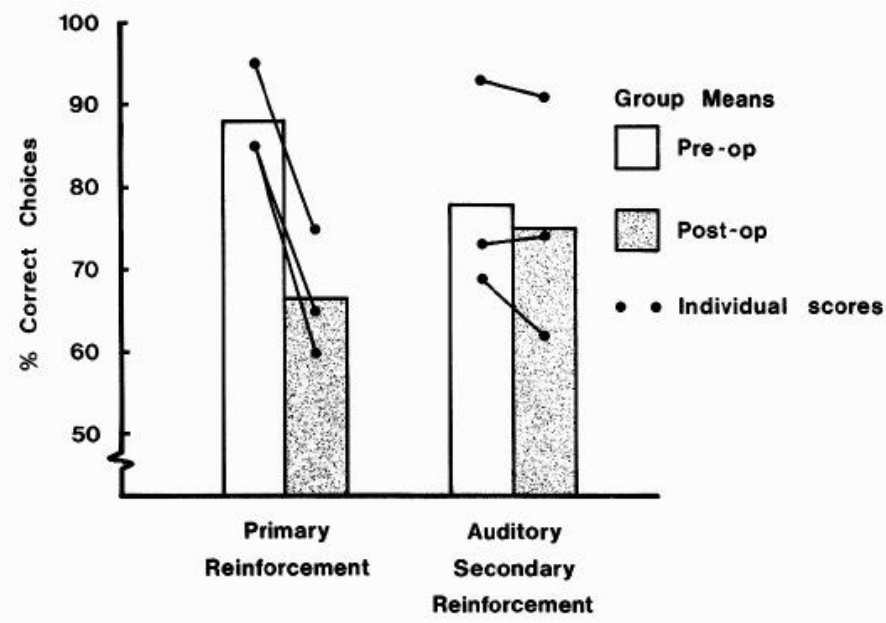

Figure 3. A comparison of the present experiment (left) with that of Gaffan and Harrison (1987; right). Both experiments examined the effect of disconnecting the amygdala from visual association cortex on visual discrimination learning. In the present experiment, the visual stimuli were associated with food reward, but in the earlier experiment they were associated with an auditory secondary reinforcer. As an indication of the efficiency of learning, the graph shows the percentage of correct choices on trial 4 of the visual discrimination problems. The same animals were tested when normal (Pre-op) and after disconnection surgery (Post-op). The bars show group averages and the lines show individual animals.

\section{Responses during 1-stimulus trials}

Behavior during the 1-stimulus presentations themselves, as distinct from the effect of those trials upon subsequent 2-stimuli choice trials, was assessed by counting the number of times the animal touched the stimulus and by accumulating the total time spent in contact with the stimulus. These 2 measures gave similar pictures. Table 2 presents the number of touches, and shows the mean number of responses to all presentations of $\mathrm{S}+$ and $\mathrm{S}-$, except the first in each problem (when differentiation was of course impossible), pooled over problem types 2-4 across the 3 surgical stages of the experiment. Two animals showed little or no sign of responding differently to $\mathrm{S}+$ and $\mathrm{S}-$, and one of them rarely touched either. One monkey, No. XVA-1, touched the $\mathrm{S}+$ stimuli more than the $\mathrm{S}-$ stimuli; this monkey continued to show some discrimination in the number of touches following the disconnection surgery. This was also the individual animal that showed the best overall performance in choice trials at all stages (Table 1). The consistent effect of the surgery on choice trials in all 3 subjects (Table 1, Fig. 3), despite the subjects' widely varying response rates during the 1 -stimulus trials (Table 2), suggests that associative learning during the 1-stimulus trials was independent of overt responses to the stimuli during the 1-stimulus trials.

\section{Discussion}

The experiment had 3 main purposes. The first was to test the hypothesis that disconnection of the inferotemporal area from the amygdala would impair monkeys' learning of associations between visual stimuli and food rewards. This hypothesis was confirmed. The second was to compare the effects of the disconnection upon stimulus-reward-associative learning under 2 acquisition conditions, one contingent upon the animals' choices 
and the other not. These 2 acquisition conditions were almost equally effective in normal animals, and were equally affected by disconnection. Thus we conclude that the amygdala participates in stimulus-reward-associative learning both when the animal chooses a visual stimulus by touching it and when a visual stimulus signals reward independently of the animal's behavior in response to that stimulus. The third purpose was to draw a comparison between the effects of disconnection upon learning of associations with food and its effects upon learning of associations with an auditory secondary reinforcer, as established in an earlier experiment (Gaffan and Harrison, 1987). The severe impairment in the present experiment, with primary food reinforcement, contrasted sharply with the absence of impairment in the previous experiment, with auditory secondary reinforcement. This contrast is further sharpened by some differences in the surgical operations in the 2 experiments. Gaffan and Harrison (1987) ablated both the inferotemporal area unilaterally and the amygdala contralateral to the inferotemporal ablation in one operation; these 2 ablations were carried out in 2 stages of surgery in the present experiment. Since one-stage surgery in general produces a greater impairment than multiplestage surgery (Finger, 1978), this difference is unlikely to explain the contrasting behavioral results. Further, Gaffan and Harrison's (1987) animals remained unimpaired when the forebrain commissures and the fornix-fimbria were transected; the impairment in the present experiment was manifest in animals with the forebrain commissures and the hippocampal system intact. For these reasons, the difference between the effects of disconnection in the 2 experiments can be ascribed to the difference in the tasks.

There are several sources of evidence in favor of the hypothesis (outlined in the introduction) that the amygdala is involved in associating stimuli with the intrinsic reward value of food. One is the well-established effects of amygdalectomy on oral behavior. The observation that amygdalectomized monkeys frequently take inedible objects into their mouths (Weiskrantz, 1956; Horel and Keating, 1969, 1972) is consistent with the hypothesis that the operation has impaired the association of the visual appearance of objects with their reward value as food. Evidence also comes from the reports that bilateral amygdalectomy retards visual discrimination learning for food reward; these reports, by Schwartzbaum and Poulos (1965) and others, were discussed in some detail by Gaffan and Harrison (1987). A third source of evidence is the experiment of Gaffan and Harrison (1987); the present experiment strengthens that evidence by ruling out an alternative interpretation of their findings in terms of response contingency, as explained in the introduction.
The effect of the crossed unilateral lesions of the amygdala and the inferotemporal cortex, in the present experiment, is to disconnect the normal direct interaction between those 2 structures: Following the unilateral lesions, one inferotemporal area remains intact and one amygdala remains intact, but the direct projection from the inferotemporal area to the amygdala (Herzog and Van Hoesen, 1976) is disrupted bilaterally by destruction of its origin in one hemisphere and destruction of its termination in the other. In earlier work on the inferotemporal area (D. Gaffan et al., 1986; E. A. Gaffan et al., 1986), we argued that the inferotemporal area provides an input to a memory system of associations with food reward. It is therefore an attractive hypothesis that the acquisition of associations between visual stimuli and the reward value of food depends upon a modification in the amygdala of the cells that receive the projection from the inferotemporal area.

\section{References}

Finger, S. (ed.) (1978) Recovery from Brain Damage: Research and Theory, Plenum, New York.

Gaffan, D., and S. Harrison (1987) Amygdalectomy and disconnection in visual learning for auditory secondary reinforcement by monkeys. J. Neurosci. 7: 2285-2292.

Gaffan, D., and S. Harrison (1988) Inferotemporal-frontal disconnection and fornix transection in visuo-motor conditional learning by monkeys. Behav. Brain Res. (in press).

Gaffan, D., R. C. Saunders, E. A. Gaffan, S. Harrison, C. Shields, and M. J. Owen (1984) Effects of fornix transection upon associative memory in monkeys: Role of the hippocampus in learned action. Q. J. Exp. Psychol. 36B: 173-221.

Gaffan, D., S. IIarrison, and E. A. Gaffan (1986) Visual identification following inferotemporal ablation in the monkey. Q. J. Exp. Psychol. $38 B: 5-30$.

Gaffan, E. A., S. Harrison, and D. Gaffan (1986) Single and concurrent discrimination learning by monkeys after lesions of inferotemporal cortex. Q. J. Exp. Psychol. 38B: 31-51.

Gamzu, E., and E. Schwam (1974) Autoshaping and automaintenance of a keypress response in squirrel monkeys. J. Exp. Anal. Behav. 21: 361-371.

Herzog, A. G., and G. W. Van Hoesen (1976) Temporal neocortical afferent connections to the amygdala in the rhesus monkey. Brain Res. 115: 57-69.

Horel, J. A., and E. G. Keating (1969) Partial Kluver-Bucy syndrome produced by cortical disconnection. Brain Res. 16: 281-284.

Horel, J. A., and E. G. Keating (1972) Recovery from a partial KluverBucy syndrome in the monkey produced by disconnection. J. Comp. Physiol. Psychol. 79: 105-114.

Schwartzbaum, J. S., and D. A. Poulos (1965) Discrimination behavior after amygdalectomy in monkeys: Learning set and discrimination reversal. J. Comp. Physiol. Psychol. 60: 320-328.

Weiskrantz, L. (1956) Behavioral changes associated with ablation of the amygdaloid complex in monkeys. J. Comp. Physiol. Psychol. 49: $381-391$. 